\title{
Commentary: Family Planning Use Among Women Living with HIV: A National Survey
}

Jane Namasasu*

Independent Consultant, Lilongwe 3, Malawi

\section{Article Info}

\section{Article Notes}

Received: December 3, 2018

Accepted: January 14, 2019

\section{*Correspondence:}

Dr. Jane Namasasu, Independent Consultant, P.O. Box 30760,

Lilongwe 3, Malawi; Email: jnamasasu@yahoo.com

${ }^{\circ} 2019$ Namasasu J. This article is distributed under the terms of the Creative Commons Attribution 4.0 International License.

\section{Original article citation:}

"Family planning use among women living with HIV knowing their HIV positive helps-results from a national survey" by Dereje Habte \& Jane Namasasu. BMC journal Reproductive health Vol.12:41, 2015. Doi:10.1186/s12978-015-0035-6

\section{Key words:}

Family planning

Women

HIV

PMTCT
There were 36.9 people living with HIV and 940,000 deaths globally in 2017 while 1.8 million were newly infected with the virus in the same year ${ }^{1}$. Available reports indicate that African region is the mostly affected with nearly 1 in 25 adults living with HIV $^{1}$. However globally, HIV related deaths are declining due to antiretroviral therapy. Nonetheless, AIDs related illness remain the leading cause of death among women of reproductive age group 15-49 years in the world and still the second leading cause of deaths in young women aged 15-24 years in Africa ${ }^{2}$. In fact the diseases progression and it's harmful effects on other pregnant-related contributes significantly to maternal mortality. AIDs is not curable but can be prevented and family planning is one of the strategies in preventing HIV and antiretroviral drugs for preventing mother to child transmission of $\mathrm{HIV}^{3}$.

In Malawi, in 2017 about one million people were living with HIV with a prevalence rate of $9.6 \%$ among adults between 15-49 years age group. New infected with the virus were 39,000 and $17 \%$ related deaths due to AIDS and $71 \%$ of adults living with HIV are on ART while $63 \%$ of children living with HIV are on ART ${ }^{4}$. Malawi has made tremendous efforts to reducing the HIV epidemic due to various strategies which were put in place such as HIV testing and counseling, HIV prevention programmes such as availability and use of condoms, HIV education and prevention of mother to child transmission (PMTCT) ${ }^{5}$. For example, a population-based HIV impact assessment conducted in Malawi in 2015 to 2016, found that $72.7 \%$ of people living with HIV (PLHIV) 15-64 years olds reported they know their HIV status these were $76.3 \%$ HIV positive females and $66.7 \%$ positive males ${ }^{6}$. The $2015-2020$ strategic plan key areas of focus are elimination of HIV with 90-90-90 targets set by UNAIDS in 2014 and targeting HIV activities in key and vulnerable populations ${ }^{7}$. The high impact interventions to reach these targets include, routine testing of HIV, prevention of mother to child transmission (PMTCT): It has four prongs, one of which is to reduce unplanned or unintended pregnancy among the HIV positive women and continue with scale up of $\mathrm{ART}^{7}$.

Habte \& Namasasu, in a survey published in BMC Reproductive Health Journal in 2015, analysed data from demographic and health survey of 2010 among 489 non-pregnant women, who are sexually active, fecund living with HIV. The aim of this analysis was to assess family planning practice among HIV infected women and the influence of their awareness of HIV positive status in the practice of family planning. The authors performed multiple logistics regression 
analysis using SPSS software to identify factors associated with family planning use. The analysis was conducted in three phases. The first phase included socio demographic variables such as age, education, marital status, religion and residence. In addition to the socio demographic variables, the second phase included family planning information from the radio, visit to a health facility, births in the past five years and number of living children. Phase three added women's knowledge of HIV status in addition to data in phase two ${ }^{8}$.

The results showed that 489 laboratory confirmed HIVinfected women who were sexually active in the one year preceding the survey were included in the analysis. Over four-fifth of the women were aged 25 years and above. Women with no formal education, primary/secondary/ post-secondary education constituted $14.9 \%, 63.8 \%$ and $21.3 \%$ respectively. The majority of women were married/ cohabiting $(71.8 \%)$, from the southern region, $(64.0 \%)$, rural residents $(74.0 \%)$ and currently working $(67.3 \%)$. These findings are consistent with a study conducted in Cameroon where women with no primary education were at $30.5 \%$ while $47.5 \%$ attended primary school level and those with secondary and tertiary education were at $18 \%$ and $4 \%$ respectively ${ }^{9}$. Women in the richer and the richest category were $22.3 \%$ and $32.1 \%$ respectively whereas the proportions of the poorest, poorer and middle categories $13.1 \%, 15.7 \%$ and $16.8 \%$. In the bivariate analysis, none of the socio-demographic variables were significantly associated with current practice of family planning ${ }^{8}$.

The proportion of women who accessed information via radio, televison and newspaper were 65\%, $14.1 \%$ and $16.6 \%$ respectively. A significant majority of the respondents said that they were not visited by a family planning worker at home in the year preceding the survey while $76.5 \%$ reported to have visited a health facility in the past one year. In the five year preceding the survey, $44.6 \%$ women did not give any birth, $38.9 \%$ had one birth and $16.6 \%$ reported two or more births. Over one-third of women did not have under-five children ${ }^{8}$.

The results further showed that those with secondary education and above were significantly associated with current use of family planning methods with adjusted odds ratio and 95\% confidence interval (AOR, 95\% CI) of 2.06 $(1.08,3.91)$ married/cohabiting women with AOR (95\% CI) of $1.51(1.00,2.26)$ and women from the central region AOR $(95 \% \mathrm{CI})$ of $0.53(0.28,0.99)$. Secondary education and above and being from the central region, maintained associations (AOR:2.17 (1.08,4.36) and $0.51(0.26,0.99)$ ). Women who had one birth and more than one birth in the past five years were more likely to practice family planning compared to the ones without births. Likewise, the number of living children was also associated with family planning use.The highest association was with women who had five living children. Similarly women who were knowledgeable of their HIV status were associated with family planning use. This is consistent with a study conducted in Ethiopia where number of children were also a factor in contraceptive use $\mathrm{e}^{10}$.

The analysis of this national survey has indicated that secondary education and above, having living children and knowledge of their HIV status are some of the predictors to using family planning methods ${ }^{9}$. This may be because education empowers men and women to gain insight and awareness of what HIV and AIDS, is all about and how it can be prevented. Additionally use of Antiretrovirus drugs among HIV positive women has made a difference in prevention of mother to child transmission (MTCT) results also noted in a study conducted in Cameroon ${ }^{9}$. If family planning methods are not used, the results would be unwanted pregnancies, vertical transmission of the virus to the unborn baby and increased maternal deaths. Therefore if all women who do not wish to get pregnant accessed contraceptive services as many as an additional 160,000 births could be averted ${ }^{11}$. Wilcher \& Cates recommends that PMTCT programmes should reach women and their partners outside of antenatal clinic before they become infected with the virus. Controlling mother to child transmission of HIV is a key priority among women of childbearing age but it is also said to be the most difficult prong to implement ${ }^{7}$. Prevention of mother to child transmission has four prongs. Prong one is prevention of primary HIV infection in women. Prong two, prevention of unintended pregnancies (Family planning) in HIV-positive ${ }^{12}$ (Magala, et al. 2017) women, prong three, prevention of transmission from HIV infected woman to her infant and four, to support mother and family (ibid). Available evidence in the literature corroborates these findings ${ }^{13,14,15,16}$.

The authors have discussed effectively the content about family planning use among women living with HIV, knowing their HIV positive status using secondary data from the Malawi Demographic Health Survey of $2010^{17}$. The aim for analysing this secondary data from the national survey was to assess family planning use among HIV infected women and level of awareness of their HIV positive status ${ }^{8}$. It was important to understand family planning use, pregnancy intentions and knowledge among HIV positive women in Malawi. Available information indicate that if women who were HIV positive were provided with adequate information and were counseled about risks of getting pregnant in their status, they would live longer life, by reducing maternal deaths and morbidity, delaying pregnancies by spacing and preventing unwanted pregnancies ${ }^{18}$. In addition, these women would take precautionally measures to prevent further HIV infection and make informed decisions about when to get pregnant ${ }^{11}$. Helping women with HIV to fulfill their desired family size and decreasing mother to child 
transmission of HIV was seen as one of the best strategies to prevent further HIV transmission as stipulated in the Malawi National Plan for Elimination of MTCT.

The authors presented in detail the challenges women living with HIV encounter, unintended pregnancies, desire to continue having children even though their babies risk contracting mother to child transmission (MCTC) of HIV, lack of information about MCTC, poor outcomes of previous pregnancies and unmet need for family planning. Family planning and HIV data have been used cohesively with supporting evidence from the literature. As has been discussed in the paper main mode of transmission of HIV is hetero-sexual intercoarse and MTCT which can be addressed by Family planning use and counseling of couples about HIV and AIDs prevention. Those women who are HIV positive can benefit from option $\mathrm{B}+$ treatment (meaning pregnant women and lactating mothers are enrolled in ART programs regardless of their CD4 count).

Some of the exposure measures to HIV were adequately articulated and analysed such as socio-demographic data, access to information, reproductive history in the past five years and knowledge of HIV status, however, the gaps still exist in terms of providing HIV testing and counseling to all expected pregnancies, ART to all possible HIV infected women and HIV testing to exposed infants. Such information is cause for concern that most of these women passing through the corridors of the health facilities are missed without being given information and counseling about HIV and the importance of using family planning methods to prevent them from get infected with HIV. This is a missed opportunity working against achieving the national set target of $90-90-90^{7}$ which the National AIDS commission developed with partners focused on meeting the target 90 90- $90^{7}$.This strategy is to identify programme and system gaps and closing those identifed gaps within five years.

Reinforcing the 2015-2020 prevention strategies will contribute to reaching the set target such as a) continued expansion of HIV treatment programmes, b) promoting family planning use among women who are HIV positive or negative and c) targeted testing for men, women and youth and other family planning methods, d) raising awareness to prevent HIV through counseling and providing information, and e) prevention of mother to child transmission of HIV (PMTCT) by increasing sites for provision of ART, such strategies may prevent unintended pregnancies, prevent new infections to couples, youths and children. Missed opportunities should also be explored to ensure information and counseling services are available at the point of contact. The need to promote safe sex and responsible sexual behaviour among men should also be emphasised. Additionally future Demographic Health Surveys to explore women living with HIV's family planning needs.
In conclusion, in this commentary it is clear that women's knowledge of their HIV positive status is a predictor to family planning use. What is required by health service providers is to realise that prevention of HIV and unwanted pregnancy are linked and activities to address both of these problems should complement each other. Raising awareness about importance of using contraceptives, testing for HIV through the hospital, clinics, print and electronic media are important avenues for providing information to the population. As it has been indicated in this commentary, family planning is the second prong of PMTCT, this shows how important family planning is to preventing HIV infection, making family planning services widely accessible and consistently available through multiple points of contact with clients and patients are critical components to controlling the HIV epidemic.

Limitation: Since data used were collected in 2010, the situation may have improved since then. There is need to conduct research where variables missed in the national survey can be developed, collected and analysed. Additionally, collecting qualitative data would increase the richness of future research and detailed knowledge of family planning use, couples communication,counseling of HIV positive or negative status, stigma and disclosure of HIV results.

\section{Acknowledgments}

I wish to sincerely thank Dereje Habte one of the authors of this paper to which this commentary is based.

\section{References}

1. WHO, 2018, HIV and AIDs fact sheet.

2. UNAIDS, 2017 State of the AIDS epidemic.

3. Wekesa E, Coast E. Contraceptive needs and use among individuals with HIV and AIDS living in the slums of Nairobi Kenya. 2015.

4. UNAIDS, Data 2018.

5. Avert: Global information and education on HIV and AIDS: HIV and AIDS in Malawi. www.avert.org

6. Ministry of Health (2015-2016), Malawi population-based HIV impact (MPHIA) assessment.

7. National AIDS Commission (2015-2020) National AIDS Commission, strategic plan.

8. Habte D, Namasasu J. Family planning use among women living with HIV Knowing HIV-positive status helps results from a National Survey. Journal of Reproductive Health. 2015.

9. Nkwabong E, Minda V, Fomulu JN. Knowledge attitudes and practices of contraception by HIV-positive women followed in a Cameroon region with high illiteracy rate A cross sectional study. 2015.

10. Alene KA, Alene KA. Contraceptive use and method preference among HIV- positive women in Amhara region Ethiopia. 2018.

11. Wilcher R, Cates W. Reproductive choices for women with HIV: Bulletin of the World Health Organisation. 2009.

12. Magala I, Onega L. Factors influencing contraceptive among sexually active HIV positive clients:The Aids supporting organization (TASO) 
Masaka Uganda Journal of Public Health Policy and planning. 2017; $1: 2$.

13. OniEE, Ross A. Contraceptive practices amongst HIV-positive women on antiretroviral therapy attending ART clinic in South Africa. 2013.

14. USAID (2013), Family planning counseling for women living with HIV: Research to Prevention. www.jhsph,edu

15. Laryea DO, Amoako YA, Spangenberg K, et al. Contraceptive use and unmet need for family planning among HIV positive women on antiretroviral therapy in Kumasi, Ghana; BMC Women's Health. 2014; 14 (1): 126.
16. Mitiku K, Mulugeta S. Modern contraceptive utilization and associated factors among HIV positive women on antiretroviral therapy in Mizan-Tepi Teaching hospital and referral hospital, SouthWest Ethiopia. Journal of Reproductive Health and Contraception. 2017.

17. Ministry of Health (2010), Malawi Demographic and Health Survey.

18. Elul B, Delvaux T, Munyana E, et al. Pregnancy desires and contraceptive knowledge and use among prevention of mother-tochild transmission clients in Rwanda. International AIDS Society Journal. 2009. 